\section{Utilização dos registros de dispensação da farmácia como indicador da não-adesão à terapia anti-retroviral em indivíduos infectados pelo HIV}

\author{
Pharmacy records as an indicator of non-adherence \\ to antiretroviral therapy by HIV-infected patients
}

\author{
1 Gerência de Epidemiologia \\ e Informação, Secretaria \\ Municipal de Saúde de Belo \\ Horizonte, Belo Horizonte, \\ Brasil. \\ 2 Faculdade de Medicina, \\ Universidade Federal de \\ Minas Gerais, Belo Horizonte, \\ Brasil. \\ 3 Centro de Desenvolvimento \\ e Planejamento Regional \\ Universidade Federal de \\ Minas Gerais, Belo Horizonte, \\ Brasil. \\ 4 Faculdade de Farmácia, \\ Universidade Federal de \\ Minas Gerais, Belo Horizonte, \\ Brasil. \\ Correspondência \\ R. R. F. M. Gomes \\ Gerência de Epidemiologia \\ e Informação, Secretaria \\ Municipal de Saúde de Belo \\ Horizonte. \\ Rua Dr. Virgílio Uchoa 627 , \\ Belo Horizonte, $M G$ \\ 30320-240, Brasil. \\ quelfmg@medicina.ufmg.br
}

\begin{abstract}
The objectives of this study were to evaluate anti-retroviral (ARV) prescription pickups during twelve months following the first prescription and to identify factors associated with irregular pickups or permanent dropout in two public HIVIAIDS referral centers in Belo Horizonte, Minas Gerais State, Brazil. Participants ( $n=323)$ were antiretroviral nä̈ve and were recruited from May 2001 to May 2002. A total of 98 (30.3\%) patients abandoned treatment, and 187 (57.9\%) had at least one irregular pickup. Patients with irregular pickups and dropouts were compared to those with regular pickups. Multinomial multivariate analysis showed that living outside Belo Horizonte, CD4+ T-lymphocyte count greater than $200 / \mathrm{mm}^{3}$, and antiretroviral regimen without protease inhibitors were associated with irregular pickups. In addition to these variables, not being on other medications, and any non-adherence recorded on patient charts were associated with treatment dropout. Pharmacy records are important potential indicators of non-adherence and should be incorporated as such in clinical practice. Strategies should be prioritized to reach out to dropouts or patients with irregular ARV pickups.
\end{abstract}

HIV; Acquired Immunodeficiency Sindrome; Anti-Retroviral Agents; Pharmacy
Raquel Regina de Freitas Magalhães Gomes 1,2 Carla Jorge Machado 3

Francisco de Assis Acurcio 2,4

Mark Drew Crosland Guimarães 2

\section{Introdução}

As políticas públicas brasileiras para a provisão de assistência à saúde de pacientes infectados pelo vírus da imunodeficiência humana (HIV) têm tido reconhecimento em âmbito mundial, principalmente a garantia do acesso universal e gratuito aos anti-retrovirais (ARV) ${ }^{1}$. Atribui-se a essas políticas parte do sucesso do tratamento, com reflexos na redução da mortalidade e internações devido ao HIV em anos recentes ${ }^{2}$. Atualmente, dos 17 tipos de ARV distribuídos, oito são produzidos por laboratórios brasileiros, o que tem proporcionado uma significativa redução dos custos. Em 2008, cerca de 200 mil pessoas infectadas pelo HIV estão em tratamento no Brasil e os gastos do Ministério da Saúde, considerando apenas estes medicamentos, representam R\$ 1 bilhão ${ }^{3}$. Entretanto, a grande dificuldade de se obter uma adequada efetividade do tratamento é o de assegurar uma boa adesão do paciente ao esquema prescrito de ARV, evitando-se assim a falência do tratamento pela utilização de doses subótimas ou de forma irregular. A sua ocorrência possibilita o desenvolvimento de cepas virais resistentes, reduzindo as opções terapêuticas disponíveis e a sobrevida do paciente, bem como o risco da transmissão destas para parceiros nãoinfectados 4

A avaliação da qualidade da assistência ambulatorial e a identificação dos possíveis fatores associados com a não-adesão do paciente à tera- 
pia tornaram-se uma prioridade. Nemes et al. 5 avaliaram três indicadores da qualidade da assistência em 322 serviços de saúde de sete estados brasileiros: disponibilidade de recursos, organização e gerenciamento técnico do trabalho. Os serviços obtiveram melhores pontuações nos indicadores relacionados à estrutura (utilização dos medicamentos, testes laboratoriais e recursos humanos), enquanto os indicadores relacionados ao processo da atenção e administração apresentaram piores pontuações indicando a necessidade de imediata intervenção nesta área.

A adesão é um processo dinâmico que, além de ser influenciado pelo contexto sócio-cultural, depende fundamentalmente da qualidade do relacionamento entre pacientes e profissionais de saúde e do modo como os indivíduos infectados pelo HIV enfrentam suas possibilidades para obter uma melhor qualidade de vida. Sendo assim, uma avaliação precisa da adesão é fundamental para um adequado planejamento do tratamento. Considera-se que, para se manter a carga viral indetectável, é necessário que pelo menos 95\% dos medicamentos ARV prescritos sejam efetivamente tomados 6,7. Com a não existência de uma medida "padrão-ouro", diferentes métodos são utilizados para estimar a não-adesão, incluindo: auto-relato (entrevista estruturada), prontuário médico, contagem de pílulas, registro diário de uso de medicamentos, verificação dos níveis plasmáticos dos ARV, sistema de monitorização eletrônica e os registros de dispensação da farmácia. Dessa forma, apresentam resultados que variam de $25 \%$ a 43,1\% de não-adesão 8,9,10,11,12,13,14 .

Desde 1997, a Organização Mundial da Saúde (OMS) juntamente com a Federação Farmacêutica Internacional têm reconhecido o papel relevante dos profissionais farmacêuticos na luta contra a epidemia da AIDS, por promover o desenvolvimento de uma boa prática de dispensação, aconselhamento individual, apoio no acompanhamento dos pacientes e na promoção da adesão 15. Além disso, diversos estudos têm ressaltado a relevância da assistência farmacêutica na promoção da adesão à terapia ARV $16,17,18,19$, melhorando a resposta virológica dos pacientes infectados 20 .

No Brasil, na 1a Conferência Nacional de Medicamentos e Assistência Farmacêutica em 2003, foram apresentadas propostas para aplicabilidade da Assistência Farmacêutica em áreas essenciais, como as DST/AIDS, que, por sua vez, apresentam impacto direto nos gastos do Ministério da Saúde com medicamentos. Nessa direção, a Atenção Farmacêutica além de ter como meta o cuidado ao usuário, também pode ser uma estratégia na promoção do uso racional dos medicamentos 21. O sistema de dispensação dos ARV segue normas padronizadas pelo Programa Nacional de DST e AIDS (PN-DST/AIDS), e somente as farmácias dos serviços de assistência especializada credenciados são autorizadas a distribuir os medicamentos, sendo denominadas de unidades dispensadoras de medicamentos. Os pacientes com indicação para iniciar a terapia ARV devem ser notificados no Sistema Nacional de Informação de Agravos de Notificação (SINAN) e cadastrados no Sistema de Controle Logístico de Medicamentos (SICLOM) da farmácia, onde são orientados em relação ao esquema prescrito. Como regra geral, a dispensação é feita mensalmente de acordo com a prescrição, quando são registrados o número de comprimidos e a dose de cada medicamento, incluindo trocas e modificações posológicas 22. Embora não seja possível afirmar se a medicação retirada da farmácia foi utilizada adequadamente, as informações sobre as dispensações dos medicamentos tornam-se importante instrumento na identificação precoce dos pacientes em risco de não-adesão. Por ser um método simples e que utiliza um sistema padronizado pelo PN-DST/AIDS, pode ser incorporado à prática clínica na orientação aos pacientes em tratamento.

A medida de adesão baseada nos registros de dispensação da farmácia tem sido mostrada como um indicador válido e confiável em estudos de associação com resultados virológicos e imunológicos 7,23,24,25, progressão clínica e mortalidade 26,27 .

Também se destaca pela oportunidade de se avaliar todos os pacientes que iniciaram terapia e que retornam mensalmente para retirar os medicamentos, sendo possível verificar a situação de cada um, as ocorrências de irregularidades e as interrupções de tratamento. Além disso, as dificuldades iniciais relatadas com o tratamento 28 podem aumentar o risco do não cumprimento da prescrição e do abandono do acompanhamento médico 29 , havendo poucos estudos brasileiros que avaliam de forma prospectiva as dispensações de ARV nesta fase do tratamento.

Assim, o objetivo principal deste estudo foi avaliar as retiradas irregulares de ARV da farmácia e os abandonos da terapia por pacientes infectados pelo HIV em início de terapia em serviços públicos de referência como indicador de não-adesão e identificar seus fatores associados.

\section{Metodologia}

\section{População e desenho}

Esta análise faz parte de um estudo maior (Projeto ATAR) 30, cujo principal objetivo foi determinar a 
incidência de adesão ao tratamento anti-retroviral em indivíduos infectados pelo HIV, utilizando diferentes medidas de não-adesão. A não-adesão foi medida pelo auto-relato 31 , pelo registro diário de uso dos medicamentos ${ }^{32}$, pelo prontuário médico 30 e pelos registros de dispensação dos ARV, objeto desta análise. Sinteticamente, o Projeto ATAR é um estudo prospectivo concorrente, no qual os pacientes foram recrutados de dois serviços públicos de referência para AIDS, o Centro de Treinamento e Referência de Doenças Infecto-Parasitárias (CTR-DIP) e o ambulatório do Hospital Eduardo de Menezes (HEM) em Belo Horizonte, Minas Gerais, entre maio de 2001 e maio de 2002, com acompanhamento até maio de 2003. Foram incluídos indivíduos comprovadamente infectados pelo HIV, em início de terapia, virgens de tratamento ARV, com idade igual ou superior a 18 anos.

Para esta análise, optou-se por excluir todos os participantes com registro de transferência e/ ou de óbito no período analisado, pela impossibilidade de se determinar quando estas ocorreram, evitando-se assim superestimar o abandono. Analogamente, foram excluídas as gestantes pelas especificidades da terapia ARV (duração e esquemas), cuja interrupção precoce pode ser devida à indicação médica.

Inicialmente, os pacientes foram entrevistados (entrevista basal) logo após receberem seus primeiros medicamentos ARV nas farmácias de cada serviço e acompanhados por um período de 12 meses com visitas de seguimento nos meses um, quatro e sete. Os registros mensais das dispensações dos ARV foram pesquisados nas farmácias dos centros participantes após o período de acompanhamento.

Formulários padronizados com manuais de preenchimento foram elaborados e testados para as entrevistas, para a coleta de dados clínicos e laboratoriais em prontuários médicos e para os registros das dispensações nas farmácias. Em seguida, foram codificados, digitados e organizados em bancos de dados. Os participantes receberam um número de ordem e de identificação que os individualizavam nos diversos bancos, garantindo o anonimato. A análise de confiabilidade realizada confirmou a qualidade das informações obtidas 30 . O projeto foi aprovado pelo Comitê de Ética em Pesquisa da Universidade Federal de Minas Gerais (COEP/UFMG; parecer ETIC 106/99) e cada participante assinou um termo de consentimento livre e esclarecido.

\section{Evento}

Foram analisadas todas as dispensações dos ARV registradas nas farmácias dos serviços mensal- mente até o 12o mês de seguimento. Para cada dispensação foram obtidas informações sobre as datas da primeira e subseqüentes dispensações, esquemas de ARV dispensados, número de comprimidos e dose de cada medicamento e ocorrência de trocas. Foi considerado como troca de esquema a inclusão, exclusão ou ajuste de dose de pelo menos um ARV.

O tempo entre as retiradas de ARV foi calculado pela diferença, em dias, entre as datas das dispensações, até o 12o mês após a prescrição. A análise do conjunto dos tempos transcorridos entre as dispensações foi necessária, com vistas a estabelecer o ponto de corte para decisão de quantos dias uma retirada seria considerada irregular. Assim, houve 3.206 intervalos de tempos transcorridos para 323 pacientes. A distribuição dos tempos apresentou-se aproximadamente normal, unimodal, com assimetria à direita, com valores no intervalo de 1 a 343 dias. A moda, mediana e média encontradas foram, respectivamente, 28, 30 e 34 dias, apresentando um desvio padrão de 20,4 dias. Assim, baseado na distribuição dos tempos entre as retiradas e no tempo mínimo esperado de 30 dias entre estas, considerou-se como irregular qualquer diferença superior a 34 dias.

As dispensações dos ARV foram avaliadas pelo tempo e pelo número de comprimidos dispensados para o consumo mensal. O número de comprimidos dispensados de cada ARV foi ajustado de acordo com a dose e esquemas prescritos e verificado para o consumo mensal. Em casos excepcionais, quando o serviço autorizava retiradas antecipadas de maiores quantidades de ARV, tanto o tempo quanto a contagem de comprimidos foram ajustados para o período mensal.

Dessa forma, as retiradas de ARV foram expressas pela prevalência no período de 12 meses, independentemente do momento de ocorrência das mesmas, e categorizadas em três grupos:

- Regular: quando não houve qualquer irregularidade nem no tempo nem na quantidade dispensada até o 12o mês de seguimento;

- Irregular: quando o tempo entre as dispensações foi maior do que 34 dias ou quando o número de comprimidos dispensados foi menor do que $95 \%$ do total de comprimidos esperado para cada esquema de ARV prescrito, até o 12o mês de seguimento. Para cada paciente foi computado o número de vezes em que o critério de retirada irregular foi preenchido, independente de quando este ocorreu;

- Abandono: quando o paciente ficou mais de 60 dias sem retirar os ARV e não mais retornou até o 12 o mês de seguimento. 


\section{Variáveis de exposição}

As variáveis de exposição foram agrupadas em:

- Características sócio-demográficas: local da pesquisa, sexo, idade, cor da pele, estado civil, local de residência, escolaridade, renda familiar no último mês, ter plano privado de saúde;

- Características comportamentais: uso de álcool, uso de tabaco e de drogas ilícitas no último mês antes da entrevista;

- Características relacionadas ao uso dos serviços: percepção da necessidade do uso de ARV, fazer uso de outra medicação diferente dos ARV, receber e compreender orientações médicas sobre os ARV, receber e compreender orientações dos farmacêuticos sobre os ARV, intervalo entre as consultas médicas;

- Características clínicas: classificação clínica inicial de acordo com a definição do Centers for Disease Control and Prevention (CDC, Estados Unidos), contagem de linfócitos TCD4+, primeiro esquema prescrito com inibidor da protease, troca de ARV no período, ter registro de não-adesão, ter registro de internação hospitalar.

As características sócio-demográficas, comportamentais e relacionadas aos serviços de saúde foram obtidas da entrevista basal e as características clínicas, dos prontuários médicos. As orientações médicas e farmacêuticas incluíam os seguintes itens: nomes dos ARV, quantidades, horários, dieta, utilização de bebida alcoólica, reações adversas, o que fazer em caso de não tomar os ARV e quando retornar para a dispensação dos ARV. Se os pacientes foram informados de pelo menos quatro dos itens descritos acima, a orientação era considerada recebida e/ou compreendida.

\section{Análise estatística}

Foi realizada uma análise descritiva por meio da distribuição de freqüência das variáveis categóricas e medidas de tendência central das variáveis contínuas. Com base nas médias das variáveis contínuas idade e escolaridade, foi selecionado o ponto de corte (menor que 35 anos e menor que oito anos de escolaridade, respectivamente) e estas variáveis foram analisadas como categóricas. As diferenças de proporção foram avaliadas pelo teste do qui-quadrado com nível de significância de 0,05.

Procedeu-se a uma análise univariada comparando indivíduos que tiveram pelo menos uma retirada irregular no período ou não mais retornaram para a retirada dos ARV (abandono) com aqueles que retiraram a medicação de forma regular, i.e., todos os meses. A magnitude das associações foi estimada pelo odds ratio (OR) com intervalo de 95\% de confiança (IC95\%). $\mathrm{O}$ efeito independente das variáveis explicativas foi avaliado por meio de regressão logística multinomial multivariada, comparando-se cada grupo (pelo menos uma retirada irregular ou abandono) com aqueles com retirada regular. Iniciou-se o modelo multivariado completo, incluindo todas as variáveis estatisticamente associadas com a retirada irregular ou com o abandono na análise univariada com valor de $\mathrm{p}<0,20$, além daquelas de interesse clínico e/ ou epidemiológico. Permaneceram no modelo final somente as variáveis associadas estatisticamente com retirada irregular e abandono, com valor de $\mathrm{p} \leq 0,05$. A adequação dos modelos logísticos foi verificada pelo teste de Hosmer-Lemeshow ${ }^{33}$. Para a análise estatística dos dados foram utilizados os programas Epi Info, versão 3.3.2 (CDC, Atlanta, Estados Unidos) e o SAS System for Windows 9.0 (SAS Inst., Cary, Estados Unidos), enquanto os dados foram armazenados em Paradox (Borland International, Scotts Valley, Estados Unidos).

\section{Resultados}

\section{Análise descritiva}

Entre os 473 pacientes recrutados e elegíveis, 406 concordaram em participar do estudo. Na ocasião da coleta dos registros das dispensações três fichas não foram localizadas, perfazendo um total de 403 fichas $(85,2 \%)$. Dentre essas, foram excluídas 58 gestantes, 13 óbitos (1 gestante) e 12 transferências (2 gestantes) que ocorreram no período, totalizando 323 participantes para esta análise. Não houve diferenças estatisticamente significativas entre participantes e não-participantes com relação ao gênero ( $p=0,164)$; idade $(p=0,111)$; gestantes $(p=0,506)$; e ter autonomia mínima $(\mathrm{p}=0,301) 30$.

Em relação às características sócio-demográficas, $81,7 \%$ dos pacientes foram atendidos no CTR-DIP, $67,2 \%$ eram do sexo masculino, $73,4 \%$ tinham cor da pele preta ou parda, $53,2 \%$ tinham menos de 35 anos, 60,7\% moravam em Belo Horizonte, $64,4 \%$ eram solteiros ou separados e 51,9\% tinham mais de 8 anos de escolaridade. Apenas 26,3\% relataram ter plano de saúde e $83,4 \%$ declararam ter renda familiar. Dentre as características comportamentais, a maioria relatou não ter feito uso de álcool $(62,8 \%)$, de tabaco $(65,7 \%)$ e de drogas ilícitas $(90,9 \%)$ no mês anterior à entrevista inicial. As características relacionadas aos serviços de saúde mostraram que quase todos os pacientes percebiam a necessidade do uso de ARV (93,7\%) e 55,8\% 
não faziam uso de medicação diferente dos ARV. Em relação à terapia $\mathrm{ARV}$, relataram ter recebido e compreendido, respectivamente, orientação médica $(85,7 \%$ e $75,5 \%)$ e farmacêutica $(87,9 \%$ e $88,1 \%)$. As características clínicas indicaram uma alta proporção de pacientes virgens de tratamento que iniciaram tardiamente a terapia ARV em estágio avançado da doença, i.e., 42,4\% com contagem de linfócitos TCD4+ menor que 200 células $/ \mathrm{mm}^{3}$ e 55,6\% sintomáticos. Por fim, $52,9 \%$ dos pacientes utilizaram esquemas sem inibidor de protease e $23,1 \%$ tinham registros de não-adesão nos prontuários médicos.

\section{Avaliação das dispensações}

Em relação aos esquemas prescritos de ARV, verificou-se que na primeira dispensação foram prescritos 24 tipos diferentes de esquemas e nas dispensações seguintes surgiram esquemas novos devido às trocas de ARV. Logo na primeira dispensação três pacientes já tiveram seus esquemas trocados e a ocorrência de trocas de ARV foi observada em todo o período, variando de $5,1 \%$ a $8,9 \%$ entre as dispensações. Durante todo o tempo de acompanhamento, foram dispensados 43 tipos diferentes de esquemas de ARV, dos quais dez estavam incompletos, pois foram dispensados faltando pelo menos um dos ARV do esquema dispensado anteriormente. As justificativas para essas observações foram registradas nas fichas como "paciente com comprimidos em casa", "uso errado do Nefavirenz", "não toma os comprimidos corretamente", "paciente em lista de espera para Efavirenz".

Os esquemas mais prescritos de ARV para os pacientes em início de terapia foram os triplos contendo dois inibidores de transcriptase reversa análogos de nucleosídeo (ITRN), o Biovir (GlaxoSmithKline) (74,3\%), composto por Zidovudina (AZT) + Lamivudina (3TC), associados a um inibidor de transcriptase reversa não-análogo de nucleosídeo (ITRNN) ou a um inibidor de protease (IP). As combinações com o Biovir mais freqüentemente prescritas incluíam, entre os ITRNN, o Efavirenz (30,7\%) e a Nevirapina (13,6\%) e, entre os IP, o Nelfinavir (23,5\%) e o Indinavir (6,5\%). Quando ocorreu a falta do Biovir (1\%), os anti-retrovirais, AZT e 3TC, foram dispensados separadamente e as quantidades ajustadas pela dose de cada um.

Durante o período de 12 meses após a primeira prescrição, houve retiradas regulares apenas para $38(11,8 \%)$ pacientes, enquanto 98 $(30,3 \%)$ foram considerados abandonos e 187 $(57,9 \%)$ tiveram retiradas irregulares. O número de retiradas irregulares dos ARV variou de um a nove neste grupo. Além disso, observa-se que, logo no início da terapia, doze pacientes $(3,7 \%)$ já haviam abandonado seu tratamento, não retornando na segunda dispensação agendada (Tabela 1). De forma semelhante, retiradas irregulares também ocorreram desde a primeira dispensação $(1,5 \%)$, apresentando maiores percentuais na sétima (33\%) e décima segunda dispensações $(39,6 \%)$ no período.

Tabela 1

Distribuição dos pacientes com retiradas regular, irregular de anti-retrovirais (ARV) e abandono da terapia de acordo com a ordem das dispensações ocorridas no período. Projeto ATAR, Belo Horizonte, Minas Gerais, Brasil, 2001-2003.

\begin{tabular}{|c|c|c|c|c|}
\hline \multirow{3}{*}{$\begin{array}{l}\text { Ordem das } \\
\text { dispensações }\end{array}$} & \multirow{3}{*}{$\begin{array}{c}\text { Total de } \\
\text { pacientes }\end{array}$} & \multicolumn{3}{|c|}{ Caracterização das retiradas de ARV } \\
\hline & & Regular & Irregular & Abandono \\
\hline & & n (\%) * & n (\%) * & $n(\%)$ * \\
\hline $1 \underline{a}$ & 323 & $318(98,5)$ & $5(1,5)$ & $0(0,0)$ \\
\hline $2 \underline{a}$ & 323 & $256(79,3)$ & $55(17,0)$ & $12(3,7)$ \\
\hline $3 \underline{a}$ & 311 & $214(68,8)$ & $89(28,6)$ & $8(2,6)$ \\
\hline $4 \underline{a}$ & 303 & $216(71,3)$ & $81(26,7)$ & $6(2,0)$ \\
\hline $5 \underline{a}$ & 297 & $204(68,7)$ & $85(28,6)$ & $8(2,7)$ \\
\hline $6 \underline{a}$ & 289 & $189(65,4)$ & $93(32,2)$ & $7(2,4)$ \\
\hline $7 \underline{a}$ & 282 & $181(64,2)$ & $93(33,0)$ & $8(2,8)$ \\
\hline $8 \underline{a}$ & 274 & $191(69,7)$ & $68(24,8)$ & $15(5,5)$ \\
\hline $9 \underline{a}$ & 259 & $176(68,0)$ & $70(27,0)$ & $13(5,0)$ \\
\hline $10 \underline{a}$ & 246 & $175(71,2)$ & $50(20,3)$ & $21(8,5)$ \\
\hline 11a & 225 & $160(71,1)$ & $65(28,9)$ & $0(0,0)$ \\
\hline $12 \underline{a}$ & 225 & $136(60,4)$ & $89(39,6)$ & $0(0,0)$ \\
\hline
\end{tabular}

* Os percentuais das três categorias foram calculados em relação ao total de pacientes em cada dispensação. 


\section{Análises univariada e multivariada}

Os resultados das análises univariadas comparando aqueles com retirada irregular ou abandono com aqueles com retirada regular, separadamente, estão apresentados na Tabela 2, indicando que retirada irregular e abandono estão estatisticamente associados com as variáveis sócio-demográficas, ser atendido no HEM $(p=0,03$ e $\mathrm{p}=0,04)$ e morar fora de Belo Horizonte $(\mathrm{p}=$ 0,004 e p = 0,02). Não ter plano de saúde e ter feito uso de álcool no último mês mostrou associação somente com abandono ( $\mathrm{p}=0,006$ e $\mathrm{p}=0,01)$. As características relacionadas com o serviço de saúde indicaram que retirada irregular e abandono estão associados com não fazer uso de outros medicamentos diferentes dos ARV $(p=0,04$ e $p=$ 0,004). Já os pacientes que tiveram consultas com intervalo $>6$ meses apresentaram uma chance quase oito vezes maior de abandonar a terapia do que os pacientes que tiveram consultas em menos de seis meses $(\mathrm{p}=0,002)$. As variáveis clínicas, ter contagem de linfócitos TCD4+ maior que 200 células $/ \mathrm{mm}^{3}$ no início da terapia e usar esquema sem IP, mostraram associação estatisticamente significativa com retirada irregular $(\mathrm{p}<0,001$ e $\mathrm{p}=0,003)$ e abandono $(\mathrm{p}=0,002$ e $p=0,003$ ), enquanto ter algum registro médico de não-adesão mostrou associação estatística somente com abandono da terapia $(\mathrm{p}<0,001)$.

Finalmente, a Tabela 3 apresenta as variáveis associadas de forma independente com a

Análise univariada de fatores selecionados comparando retirada irregular dos anti-retrovirais (ARV) e abandono da terapia com retirada regular no período de 12 meses. Projeto ATAR, Belo Horizonte, Minas Gerais, Brasil, 2001-2003 ( $n=323$ ).

\begin{tabular}{|c|c|c|c|c|c|c|c|c|}
\hline \multirow[t]{3}{*}{ Características } & \multirow{3}{*}{$\begin{array}{l}\text { Total } \\
\text { n (\%) }\end{array}$} & \multicolumn{7}{|c|}{ Retirada } \\
\hline & & \multirow{2}{*}{$\begin{array}{l}\text { Regular } \\
\text { n (\%) * }\end{array}$} & \multicolumn{3}{|c|}{ Irregular } & \multicolumn{3}{|c|}{ Abandono da terapia } \\
\hline & & & $\mathrm{n}(\%)$ * & OR $($ IC95\%) ** & Valor de $p^{\star \star *}$ & $n(\%)$ * & OR (IC95\%) ** & 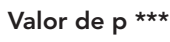 \\
\hline \multicolumn{9}{|l|}{ Sócio-demográficas } \\
\hline \multicolumn{9}{|l|}{ Local da pesquisa } \\
\hline CTR-DIP & $264(81,7)$ & $36(13,6)$ & $149(56,4)$ & 1,00 & 4,90 & $79(29,9)$ & 1,00 & 4,18 \\
\hline HEM & $59(18,3)$ & $2(3,4)$ & $38(64,4)$ & $4,59(1,06-19,92)$ & 0,027 & $19(32,2)$ & $4,33(0,96-19,58)$ & 0,04 \\
\hline \multicolumn{9}{|l|}{ Sexo } \\
\hline Masculino & $217(67,2)$ & $27(12,4)$ & $122(56,2)$ & 1,00 & 0,48 & $68(31,3)$ & 1,00 & 0,04 \\
\hline Feminino & $106(32,8)$ & $11(10,4)$ & $65(61,3)$ & $1,31(0,61-2,80)$ & 0,49 & $30(28,3)$ & $1,08(0,48-2,46)$ & 0,85 \\
\hline \multicolumn{9}{|l|}{ Idade (anos) } \\
\hline$\geq 35$ & $151(46,8)$ & $21(13,9)$ & $93(61,6)$ & 1,00 & 0,39 & $37(24,5)$ & 1,00 & 3,43 \\
\hline$<35$ & $172(53,2)$ & $17(9,9)$ & $94(54,6)$ & $1,25(0,62-2,52)$ & 0,53 & $61(35,5)$ & $2,04(0,94-4,35)$ & 0,06 \\
\hline \multicolumn{9}{|l|}{ Cor da pele } \\
\hline Branca & $83(26,6)$ & $11(13,3)$ & $49(59,0)$ & 1,00 & 0,08 & $23(27,7)$ & 1,00 & 0,47 \\
\hline Preta/Parda & $229(73,4)$ & $26(11,3)$ & $130(56,8)$ & $1,12(0,52-2,44)$ & 0,77 & $73(31,9)$ & $1,34(0,58-3,13)$ & 0,49 \\
\hline \multicolumn{9}{|l|}{ Estado civil } \\
\hline Solteiro/Separado & $208(64,4)$ & $26(12,5)$ & $124(59,6)$ & 1,00 & 0,06 & $58(27,9)$ & 1,00 & 0,99 \\
\hline Casado/União livre & $115(35,6)$ & $12(10,4)$ & $63(54,8)$ & $1,10(0,52-2,33)$ & 0,80 & $40(34,8)$ & $1,49(0,68-3,31)$ & 0,32 \\
\hline \multicolumn{9}{|l|}{ Local de residência } \\
\hline Belo Horizonte & $196(60,7)$ & $31(15,8)$ & $106(54,1)$ & 1,00 & 8,22 & $59(30,1)$ & 1,00 & 5,59 \\
\hline Fora de Belo Horizonte & $127(39,3)$ & $7(5,5)$ & $81(63,8)$ & $3,38(1,42-8,08)$ & 0,004 & $39(30,7)$ & $2,93(1,17-7,31)$ & 0,02 \\
\hline \multicolumn{9}{|l|}{ Escolaridade (anos) } \\
\hline$\geq 8$ & $167(51,9)$ & $20(12,0)$ & $105(62,9)$ & 1,00 & 0,16 & $42(25,1)$ & 1,00 & 0,96 \\
\hline$<8$ & $155(48,1)$ & $18(11,6)$ & $82(52,9)$ & $0,87(0,43-1,75)$ & 0,69 & $55(35,5)$ & $1,46(0,69-3,09)$ & 0,33 \\
\hline \multicolumn{9}{|l|}{ Renda familiar } \\
\hline $\operatorname{Sim}$ & $266(83,4)$ & $33(12,4)$ & $162(60,9)$ & 1,00 & 0,12 & $71(26,7)$ & 1,00 & 3,63 \\
\hline Não & $53(16,6)$ & $4(7,5)$ & $24(45,3)$ & $1,22(0,40-3,76)$ & 0,73 & $25(47,2)$ & $2,90(0,94-9,02)$ & 0,06 \\
\hline \multicolumn{9}{|l|}{ Plano de saúde } \\
\hline Sim & $85(26,3)$ & $15(17,6)$ & $53(62,4)$ & 1,00 & 1,86 & $17(20,0)$ & 1,00 & 7,45 \\
\hline Não & $238(73,7)$ & $23(9,7)$ & $134(56,3)$ & $1,65(0,80-3,40)$ & 0,17 & $81(34,0)$ & $3,11(1,35-7,16)$ & 0,006 \\
\hline
\end{tabular}

(continua) 


\begin{tabular}{|c|c|c|c|c|c|c|c|c|}
\hline \multirow[t]{3}{*}{ Características } & \multirow{3}{*}{$\begin{array}{l}\text { Total } \\
\mathrm{n}(\%)\end{array}$} & \multicolumn{7}{|c|}{ Retirada } \\
\hline & & \multirow{2}{*}{$\begin{array}{l}\text { Regular } \\
\mathrm{n}(\%) \text { * }\end{array}$} & \multicolumn{3}{|c|}{ Irregular } & \multicolumn{3}{|c|}{ Abandono da terapia } \\
\hline & & & $\mathrm{n}(\%)$ * & OR (IC95\%) ** V & Valor de $p * \star \star$ & $n(\%)$ * & OR (IC95\%) ** & Valor de $p * \star \star$ \\
\hline \multicolumn{9}{|l|}{ Comportamentais } \\
\hline \multicolumn{9}{|l|}{ Uso de álcool no último mês } \\
\hline Não & $194(62,8)$ & $29(14,9)$ & $115(59,3)$ & 1,00 & 1,60 & $50(25,8)$ & 1,00 & 6,61 \\
\hline Sim & $115(37,2)$ & $9(7,8)$ & $60(52,2)$ & $1,68(0,75-3,78)$ & 0,21 & $46(40,0)$ & $2,96(1,27-6,92)$ & 0,01 \\
\hline \multicolumn{9}{|c|}{ Uso de tabaco no último mês } \\
\hline Não & $203(65,7)$ & $29(14,3)$ & $113(55,7)$ & 1,00 & 1,94 & $61(30,0)$ & 1,00 & 2,01 \\
\hline $\operatorname{Sim}$ & $106(34,3)$ & $9(8,5)$ & $62(58,5)$ & $1,77(0,79-3,97)$ & 0,16 & $35(33,0)$ & $1,85(0,79-4,35)$ & 0,16 \\
\hline \multicolumn{9}{|l|}{ Uso de drogas ilícitas } \\
\hline \multicolumn{9}{|l|}{ no último mês } \\
\hline Não & $281(90,9)$ & $34(12,1)$ & $161(57,3)$ & 1,00 & 0,26 & $86(30,6)$ & 1,00 & 0,0003 \\
\hline Sim & $28(9,1)$ & $4(14,3)$ & $14(50,0)$ & $0,74(0,23-2,38)$ & 0,61 & $10(35,7)$ & $0,99(0,29-3,37)$ & 0,99 \\
\hline \multicolumn{9}{|l|}{ Serviços de saúde } \\
\hline \multicolumn{9}{|l|}{ Percepção da necessidade } \\
\hline \multicolumn{9}{|l|}{ do uso de ARV } \\
\hline Necessário & $284(93,7)$ & $35(12,3)$ & $164(57,8)$ & 1,00 & 0,13 & $85(29,9)$ & 1,00 & 0,85 \\
\hline Desnecessário/Não sabe & $19(6,3)$ & $2(10,6)$ & $7(36,8)$ & $0,75(0,15-3,75)$ & 0,72 & $10(52,6)$ & $2,06(0,43-9,88)$ & 0,36 \\
\hline \multicolumn{9}{|l|}{ Faz uso de outros } \\
\hline \multicolumn{9}{|l|}{ medicamentos } \\
\hline Sim & $137(44,2)$ & $24(17,5)$ & $79(57,7)$ & 1,00 & 4,18 & $34(24,8)$ & 1,00 & 8,53 \\
\hline Não & $173(55,8)$ & $14(8,1)$ & $97(56,1)$ & $2,10(1,02-4,34)$ & 0,04 & $62(35,8)$ & $3,13(1,43-6,83)$ & 0,004 \\
\hline \multicolumn{9}{|l|}{ Recebeu orientações } \\
\hline médicas sobre os ARV & & & & & & & & \\
\hline Sim & $264(85,7)$ & $32(12,1)$ & $154(58,3)$ & 1,00 & 0,14 & $78(29,5)$ & 1,00 & 0,007 \\
\hline Não & $44(14,3)$ & $6(13,6)$ & $24(54,5)$ & $0,83(0,31-2,20)$ & 0,71 & $14(31,8)$ & $0.96(0,34-2,71)$ & 0,93 \\
\hline Compreendeu orientações & & & & & & & & \\
\hline médicas & & & & & & & & \\
\hline $\operatorname{Sim}$ & $231(75,5)$ & $30(13,0)$ & $129(55,8)$ & 1,00 & 1,08 & $72(31,2)$ & 1,00 & 0,13 \\
\hline Não & $75(24,5)$ & $7(9,3)$ & $48(64,0)$ & $1,59(0,66-3,87)$ & 0,30 & $20(26,7)$ & $1,19(0,46-3,11)$ & 0,72 \\
\hline Recebeu orientações & & & & & & & & \\
\hline farmacêuticas sobre os ARV & & & & & & & & \\
\hline Sim & $239(87,9)$ & $31(13,0)$ & $139(58,1)$ & 1,00 & 2,63 & $69(28,9)$ & 1,00 & 2,70 \\
\hline Não & $33(12,1)$ & $1(3,0)$ & $21(63,7)$ & $4,68(0,61-36,15)$ & 0,11 & $11(33,3)$ & $4,94(0,61-39,98)$ & 0,10 \\
\hline $\begin{array}{l}\text { Compreendeu orientações } \\
\text { farmacêuticas }\end{array}$ & & & & & & & & \\
\hline Sim & $260(88,1)$ & $33(12,7)$ & $152(58,5)$ & 1,00 & 0,17 & $75(28,8)$ & 1,00 & 1,19 \\
\hline Não & $35(11,9)$ & $3(8,6)$ & $18(51,4)$ & $1,30(0,36-4,68)$ & 0,68 & $14(40,0)$ & $2,05(0,55-7,63)$ & 0,27 \\
\hline Intervalo entre & & & & & & & & \\
\hline consultas (meses) & & & & & & & & \\
\hline$<6$ & $260(82,5)$ & $35(13,5)$ & $160(61,5)$ & 1,00 & 1,73 & $65(25,0)$ & 1,00 & 9,52 \\
\hline$>6$ & $55(17,5)$ & $2(3,6)$ & $24(43,6)$ & $2,63(0,59-11,63)$ & 0,19 & $29(52,7)$ & $7,81(1,76-34,67)$ & 0,002 \\
\hline Clínicas & & & & & & & & \\
\hline Classificação & & & & & & & & \\
\hline clínica inicial (CDC) & & & & & & & & \\
\hline Sintomático (B/C) & $169(55,6)$ & $24(14,2)$ & $92(54,4)$ & 1,00 & 2,71 & $53(31,4)$ & 1,00 & 0,65 \\
\hline Assintomático (A) & $135(44,4)$ & $12(8,9)$ & $86(63,7)$ & $1,87(0,88-3,97)$ & 0,10 & $37(27,4)$ & $1,40(0,62-3,14)$ & 0,42 \\
\hline Contagem de linfócitos & & & & & & & & \\
\hline TCD4+ inicial (células/mm3) & & & & & & & & \\
\hline$<200$ & $137(42,4)$ & $26(19,0)$ & $73(53,3)$ & 1,00 & 11,07 & $38(27,7)$ & 1,00 & 9,66 \\
\hline$>200$ & $186(57,6)$ & $12(6,5)$ & $114(61,3)$ & $3,38(1,61-7,12)$ & 0,0009 & $60(32,2)$ & $3,42(1,54-7,58)$ & 0,002 \\
\hline Esquema com inibidor & & & & & & & & \\
\hline de protease & & & & & & & & \\
\hline Sim & $152(47,1)$ & $27(17,8)$ & $83(54,6)$ & 1,00 & 8,99 & $42(27,6)$ & 1,00 & 8,71 \\
\hline Não & $171(52,9)$ & $11(6,4)$ & $104(60,8)$ & $3,08(1,44-6,56)$ & 0,003 & $56(32,7)$ & $3,27(1,46-7,34)$ & 0,003 \\
\hline
\end{tabular}

(continua) 
Tabela 2 (continuação)

\begin{tabular}{|c|c|c|c|c|c|c|c|c|}
\hline \multirow[t]{3}{*}{ Características } & \multirow{3}{*}{$\begin{array}{l}\text { Total } \\
\text { n (\%) }\end{array}$} & \multicolumn{7}{|c|}{ Retirada } \\
\hline & & \multirow{2}{*}{$\begin{array}{l}\text { Regular } \\
n(\%) \text { * }\end{array}$} & \multicolumn{3}{|c|}{ Irregular } & \multicolumn{3}{|c|}{ Abandono da terapia } \\
\hline & & & $n(\%)$ * & OR (IC95\%) ** & Valor de $p$ *** & $n(\%) *$ & OR (IC95\%) ** & Valor de $p * \star \star$ \\
\hline \multicolumn{9}{|c|}{ Troca de anti-retrovirais } \\
\hline \multicolumn{9}{|c|}{ no período } \\
\hline Não & $236(75,9)$ & $30(12,7)$ & $144(61,0)$ & 1,00 & 0,33 & $62(26,3)$ & 1,00 & 3,52 \\
\hline $\operatorname{Sim}$ & $75(24,1)$ & $6(8,0)$ & $38(50,7)$ & $1,32(0,51-3,4)$ & 0,57 & $31(41,3)$ & $2,50(0,94-6,64)$ & 0,06 \\
\hline \multicolumn{9}{|c|}{ Teve registro de não-adesão } \\
\hline \multicolumn{9}{|c|}{ no prontuário } \\
\hline Não & $239(76,9)$ & $32(13,4)$ & $154(64,4)$ & 1,00 & 0,44 & $53(22,2)$ & 1,00 & 11,75 \\
\hline Sim & $72(23,1)$ & $4(5,5)$ & $28(38,9)$ & $1,45(0,48-4,43)$ & 0,51 & $40(55,6)$ & $6,04(1,97-18,46)$ & 0,0006 \\
\hline \multicolumn{9}{|c|}{ Teve registro de internação } \\
\hline \multicolumn{9}{|c|}{ hospitalar no prontuário } \\
\hline Não & $258(83,0)$ & $29(11,2)$ & $157(60,9)$ & 1,00 & 0,78 & $72(27,9)$ & 1,00 & 0,15 \\
\hline Sim & $53(17,0)$ & $7(13,2)$ & $25(47,2)$ & $0,66(0,26-1,67)$ & 0,38 & $21(39,6)$ & $1,21(0,46-3,15)$ & 0,70 \\
\hline
\end{tabular}

CTR-DIP: Centro de Treinamento e Referência de Doenças Infecto-Parasitárias; HEM: Hospital Eduardo de Menezes.

* Proporção em relação ao total para cada categoria de exposição;

** Odds ratio (OR) e intervalo de $95 \%$ de confiança (IC95\%), comparando o grupo de irregular ou abandono com aqueles com retirada regular;

*** Teste qui-quadrado.

Tabela 3

Análise multivariada multinomial * de fatores selecionados com retirada irregular dos anti-retrovirais (ARV) e abandono da terapia no período de 12 meses. Projeto ATAR, Belo Horizonte, Minas Gerais, Brasil, 2001-2003.

\begin{tabular}{|c|c|c|c|c|}
\hline \multirow[t]{2}{*}{ Variáveis } & \multicolumn{2}{|c|}{ Retirada irregular $\star \star$} & \multicolumn{2}{|c|}{ Abandono da terapia $* \star *$} \\
\hline & OR (IC95\%) & Valor de $p$ & OR (IC95\%) & Valor de $p$ \\
\hline Morar fora de Belo Horizonte & $3,21(1,31-7,87)$ & 0,01 & $2,91(1,01-8,43)$ & 0,049 \\
\hline Não fazer uso de outro medicamento & - & - & $4,61(1,77-11,97)$ & 0,002 \\
\hline Contagem de linfócitos TCD4 > 200 células $/ \mathrm{mm}^{3}$ & $2,97(1,38-6,41)$ & 0,006 & $2,60(1,0-6,79)$ & 0,051 \\
\hline Esquema sem inibidor de protease & $2,76(1,26-6,06)$ & 0,01 & $3,74(1,42-9,88)$ & 0,008 \\
\hline Ter registro de não-adesão no prontuário médico & - & - & $6,39(1,88-21,75)$ & 0,003 \\
\hline
\end{tabular}

* Retiradas irregulares ou abandono comparadas àqueles com retirada regular;

** Teste de Hosmer e Lemeshow: $\chi^{2}=1,17 ; p=0,98$;

*** Teste de Hosmer e Lemeshow: $\chi^{2}=7,54 ; p=0,48$.

retirada irregular dos ARV ou abandono da terapia. Morar fora de Belo Horizonte $(p=0,01)$, ter contagem de linfócitos TCD4+ maior que 200 células $/ \mathrm{mm}^{3}(\mathrm{p}=0,006)$ e fazer uso de esquema sem IP $(p=0,01)$ foram associados com retirada irregular de ARV, enquanto o abandono, além das variáveis citadas, também mostrou associação estatística com não fazer uso de outra medicação ( $p=0,002)$ e ter registro de não-adesão no prontuário médico $(\mathrm{p}=0,003)$.

\section{Discussão}

Os resultados desta análise indicam uma preocupante situação desses serviços públicos de referência com relação à regularidade das dispensações de ARV. São altas as proporções de retirada irregular $(57,9 \%)$ e de abandono $(30,3 \%)$, considerando-se os critérios adotados e o período analisado. O Projeto ATAR, no qual se integra este trabalho, foi o primeiro estudo de coorte no Brasil a avaliar a não-adesão dos pacientes infectados pelo HIV em início de terapia. Para efeitos comparativos, as demais medidas de não-adesão nesta mesma população foram menores. Encon- 
trou-se uma incidência acumulada de não-adesão avaliada por meio do auto-relato de $36,9 \% 31$, enquanto o registro diário de uso dos medicamentos indicou uma proporção de $25,5 \% 32$ e o registro de algum episódio de não-adesão no prontuário de $22,9 \% 30$. Questões metodológicas como definição do evento, tempo em que a medida foi obtida e a forma de coleta das informações tornam essa comparação direta impossível. São medidas distintas que podem refletir diferentes contextos da atenção ao paciente em tratamento nesses serviços. No entanto, os registros da farmácia mostraram-se adequados para detectar os pacientes em risco de efetivamente não tomar suas medicações corretamente ou mesmo de abandonar o tratamento. Além disso, alguns estudos consideram o auto-relato uma medida menos sensível para avaliar a não-adesão do que a medida baseada na retirada de medicamentos da farmácia. Enquanto Grossberg et al. 23 verificaram que pacientes $100 \%$ aderentes pelo auto-relato quando reclassificados pela medida da farmácia eram $41 \%$ não-aderentes e tinham uma resposta virológica significativamente mais baixa, Farley et al. 13 identificaram cerca de duas vezes mais indivíduos não-aderentes pelos registros da farmácia quando comparados com o auto-relato. Os registros da farmácia podem ser um importante marcador de utilização dos serviços de referência como indicador indireto para identificar pacientes em risco de não-adesão.

A taxa de abandono da terapia encontrada neste estudo foi próxima das descritas por outros autores no Brasil, que também avaliaram os registros da farmácia. Apesar da variabilidade quanto à definição do evento, Bomtempo $34 \mathrm{e}$ Brito et al. 35 encontraram taxas de interrupção da terapia de $25,2 \%$ e $35,9 \%$, respectivamente. Entretanto, as taxas de retiradas irregulares ou de atraso em buscar os ARV encontradas em outros estudos apresentaram variações de 18,9\% a $43,2 \% 14,34,36$.

Os dois serviços públicos participantes deste estudo foram responsáveis por aproximadamente $90 \%$ das notificações de AIDS no Município de Belo Horizonte no período. A associação encontrada entre ser atendido em um dos centros com retirada irregular e abandono se deve provavelmente ao fato deste serviço atender a uma proporção maior de pacientes que mora fora do Município de Belo Horizonte, cujo percentual de participantes neste estudo foi de aproximadamente $40 \%$. Por outro lado, os pacientes que moram fora da cidade e abandonaram a terapia podem ter se vinculado a outro serviço de saúde, mais próximo de suas residências e não terem propriamente abandonado o tratamento. Apesar dos pacientes sabidamente com trans- ferência terem sido excluídos da análise, esta comunicação pode não ter ocorrido para todos os casos. De qualquer forma, esse achado vem reforçar a necessidade da descentralização da rede de assistência para municípios do interior, o que favorece o diagnóstico precoce da infecção, a oportunidade de iniciar o tratamento na fase assintomática e o adequado acompanhamento da terapia pelas equipes de saúde 37 .

$\mathrm{Na}$ análise univariada, as variáveis uso de álcool no último mês e intervalo entre consultas maior que seis meses apresentaram associação estatística significativa com o abandono ( $p=0,01$ e p $=0,002$, respectivamente). Esses achados são importantes e não podem ser subestimados, embora não tenham permanecido no modelo final. A relação entre o uso de álcool e menor adesão ao tratamento já é conhecida 31,38,39. A variável intervalo entre consultas maior que seis meses, relacionada aos serviços de saúde, aponta para o acompanhamento clínico inadequado pelo serviço, apresentando em nosso estudo uma maior chance de abandono do tratamento $(\mathrm{OR}=7,81$ ). Salienta-se a importância da responsabilização dos serviços na busca do paciente faltoso, no agendamento, no acompanhamento, no aconselhamento e no acesso de todos ao serviço de saúde de modo oportuno.

Os pacientes que faziam uso de esquemas sem inibidor de protease mostraram associação estatística com retirada irregular e abandono da terapia. Cumpre ressaltar que esquemas sem IP são, em geral, de posologia mais simples e apresentam menor número de eventos adversos, o que provavelmente facilitaria a adesão ao tratamento. Entretanto, a associação encontrada poderia advir do fato de que pacientes que utilizam esquemas mais complexos geralmente recebem mais orientações dos profissionais de saúde. Da mesma forma, não fazer uso de outro medicamento diferente dos ARV mostrou associação com abandono da terapia. Os pacientes que utilizam outros medicamentos diferentes dos ARV recebem mais atenção dos profissionais dos serviços de saúde, tanto nas consultas médicas e de enfermagem quanto nas orientações dos farmacêuticos. Em razão disso, pressupõe-se que ficam mais motivados em seguir adequadamente o tratamento prescrito.

Indivíduos com contagem de linfócitos TCD4+ maior que 200 células $/ \mathrm{mm}^{3}$ tiveram uma maior chance de abandono do tratamento. A não percepção da gravidade da doença pelo paciente ausente de sintomas pode levar ao sentimento de não necessidade da medicação. Esse fato foi observado por Singh et al. 40 que relataram que a presença de infecção oportunista era um preditor positivo da adesão. Estudo qualitativo 
realizado no Brasil abordando a utilização de medicamentos por indivíduos HIV+ identificou como problema o adiamento e a recusa do cumprimento da prescrição inicial de ARV devido à ausência de sinais e sintomas da doença ${ }^{41}$. Outro estudo desenvolvido com o objetivo de determinar os fatores associados à interrupção do acompanhamento clínico ambulatorial dos pacientes com infecção pelo HIV mostrou que os indivíduos mais saudáveis tendem a utilizar menos os serviços de saúde, apresentando maior risco de abandonar a terapia 29 .

A associação entre ter tido registro de nãoadesão no prontuário médico e abandono é preocupante. Provavelmente não houve qualquer intervenção dos serviços para que esses abandonos pudessem ser evitados. Fica evidente a necessidade de mudança do processo de trabalho vigente nos serviços de referência, na tentativa de se buscar os pacientes faltosos e de se conquistar uma maior efetividade do programa de assistência aos portadores do HIV.

De qualquer maneira, é fundamental a importância dos serviços de saúde na determinação da adesão dos pacientes em início de terapia. A equipe multiprofissional deve envidar esforços de acolhimento e adesão não somente àqueles que apresentam um quadro clínico de maior gravidade, mas também aos pacientes assintomáticos, uma vez que apresentaram neste estudo maiores chances de abandonar a terapia logo no início do tratamento. Um estudo qualitativo, realizado com profissionais da farmácia de 15 unidades assistenciais de DST e AIDS concluiu que a farmácia é um espaço potencialmente favorável para escuta e construção de vínculos de confiança com os usuários. Porém, relatam a importância do reconhecimento por parte dos outros profissionais e da instituição sobre o trabalho da farmácia 42 .

Finalmente, em estudos de avaliação da adesão é importante considerar sempre os padrões metodológicos definidos, tais como os pontos de corte e os critérios de não-adesão, pois podem subestimar ou superestimar a adesão do paciente à terapia. Neste estudo, optou-se pela utilização do valor da média dos tempos observados entre as dispensações de ARV como ponto de corte para retirada irregular (34 dias). Note-se que foi superior à moda (28 dias) e à mediana (30 dias), revelando uma tolerância em torno de quatro dias. Considerou-se, assim, intervalos maiores e independentes do paciente, tais como finais de semana e feriados. Apesar do rigor estatístico adotado, os resultados são potencialmente sensíveis ao ponto de corte considerado.

Conclui-se que embora os serviços tenham uma equipe multidisciplinar, é necessário que a linha de conduta e a metodologia utilizada por todos sejam comuns e compartilhadas, para que o usuário tenha de fato uma atenção integral. É necessário o acolhimento e o acompanhamento sistemático do paciente no início da terapia por toda a equipe. Uma contribuição valiosa para isso consiste na implantação e na efetiva utilização do SICLOM pelas unidades dispensadoras dos serviços de referência, o que permite, pela facilidade de acesso às informações, monitorar e avaliar continuamente as dispensações dos ARV, com identificação rápida dos pacientes com retirada irregular ou em risco de abandono do tratamento, desta forma complementando as demais maneiras de avaliar a não-adesão ao tratamento e reduzindo o impacto da não-adesão aos ARV nos serviços públicos de referência e suas conseqüências. 


\section{Resumo}

Este estudo teve como objetivos avaliar os registros de dispensação de anti-retrovirais (ARV) por um período de 12 meses após a primeira prescrição e determinar os fatores associados com a retirada irregular ou abandono em dois serviços públicos de referência para AIDS, em Belo Horizonte, Minas Gerais, Brasil. Participaram 323 pacientes infectados pelo HIV, virgens de tratamento, recrutados entre maio de 2001 e maio de 2002. No período, 98 (30,3\%) pacientes abandonaram a terapia e 187 (57,9\%) tiveram pelo menos uma retirada irregular. Indivíduos com retirada irregular ou que abandonaram a terapia foram comparados àqueles com retirada regular. Análise multivariada multinomial indicou que morar fora de Belo Horizonte, ter contagem de linfócitos TCD4+ maior que 200 células $/ \mathrm{mm}^{3} \mathrm{e}$ uso de esquema sem inibidor de protease estavam associados com retirada irregular. Além dessas variáveis, o abandono mostrou associação com não fazer uso de outra medicação e ter registro de não-adesão no prontuário médico. Os registros da farmácia destacaram-se como potencial indicador de não-adesão, devendo ser incorporados à prática clínica. Ações que busquem os pacientes ausentes ou com retirada irregular devem ser priorizadas.

HIV; Síndrome de Imunodeficiência Adquirida; AntiRetrovirais; Farmácia

\section{Referências}

1. Okie S. Fighting HIV - lessons from Brazil. N Engl J Med 2006; 354:1977-81.

2. Dourado I, Veras MASM, Barreira D, Brito AM. Tendências da epidemia de AIDS no Brasil após a terapia anti-retroviral. Rev Saúde Pública 2006; 40 Suppl:18-22.

3. Ministério da Saúde. Notícias. Efavirenz será produzido no Brasil. http://www.saude.gov.br (acessado em 17/Set/2008).

4. Programa Nacional de DST e AIDS, Secretaria de Vigilância em Saúde, Ministério da Saúde. Recomendações para terapia anti-retroviral em adultos e adolescentes infectados pelo HIV. Brasília: Ministério da Saúde; 2006

5. Nemes MIB, Castanheira ERL, Melchior R, Alves MTSSB, Basso CR. Avaliação da qualidade da assistência no programa de AIDS: questões para a investigação em serviços de saúde no Brasil. Cad Saúde Pública 2004; 20 Suppl 2:S310-21.

6. Paterson DL, Swindells S, Mohr J, Brester M, Vergis EN, Squier C, et al. Adherence to protease inhibitor therapy and outcomes in patients with HIV infection. Ann Intern Med 2000; 133:21-30.

7. Gross R, Yip B, Lo Re 3rd V, Wood E, Alexander CS, Harrigan PR, et al. A simple, dynamic measure of antiretroviral therapy adherence predicts failure to maintain HIV-1 suppression. J Infect Dis 2006; 194:1108-14

8. Liu H, Golin CE, Miller LG, Hays RD, Beck CK, Sanandaji S, et al. A comparison study of multiple measures of adherence to HIV protease inhibitors. Ann Intern Med 2001; 134:968-77.

\section{Colaboradores}

R. R. F. M. Gomes participou da elaboração de todas as seções do artigo, realizando a revisão da literatura, análise dos dados, interpretação, discussão e redação. M. D. C. Guimarães participou da análise, interpretação, orientação e revisão do artigo. C. J. Machado participou da análise estatística e da revisão do artigo. F. A. Acurcio participou da revisão do artigo.

\section{Agradecimentos}

Este estudo foi conduzido pelo Grupo de Pesquisas em Epidemiologia e Avaliação em Saúde (GPEAS) do Departamento de Medicina Preventiva e Social da Faculdade de Medicina da Universidade Federal de Minas Gerais, em colaboração com a Faculdade de Farmácia e a Escola de Enfermagem e com o apoio financeiro do Programa Nacional de DST e AIDS, Ministério da Saúde (UNESCO 914 BRA 3014).

9. Lignani Júnior L, Greco DB, Carneiro M. Avaliação da aderência aos anti-retrovirais em pacientes com infecção pelo HIV/AIDS. Rev Saúde Pública 2001; 35:495-501.

10. Hugen PWH, Langebeek N, Burger DM, Zomer B, van Leusen R, Schuurman R, et al. Assessment of adherence to HIV protease inhibitors: comparison and combination of various methods, including MEMS, patient and nurse report, and therapeutic drug monitoring. J Acquir Immune Defic Syndr 2002; 30:324-34.

11. Pinheiro CAT, Carvalho-Leite JC, Drachler ML, Silveira VL. Factors associated with adherence to antiretroviral therapy in HIV/Aids patients: a crosssectional study in Southern Brazil. Braz J Med Biol Res 2002; 35:1173-81.

12. Murri R, Ammassari A, Trotta MP, De Luca A, Melzi S, Minard C, et al. Patient-reported and physicianestimated adherence to HAART. J Gen Intern Med 2004; 19: 1104-10.

13. Farley CK, Permana A, Read TRH. Long-term utility of measuring adherence by self-report compared with pharmacy record in a routine clinic setting. HIV Med 2005; 6:366-9.

14. Seguy N, Diaz T, Pereira-Campos D, Veloso VG, Grinsztejin B, Teixeira L, et al. Evaluation of the consistency of refills for antiretroviral medications in two hospitals in the state of Rio de Janeiro, Brazil. AIDS Care 2007; 19:617-25.

15. Commonwealth Pharmaceutical Association. The role of the pharmacist in the prevention and management of HIV/AIDS. Pharm J 2003; 271:783-4. 
16. Geletko SM, Poulakos MN. Pharmaceutical services in an HIV clinic. Am J Health Syst Pharm 2002; 59:709-13.

17. Ceccato MGB, Acurcio FA, Bonolo PF, Rocha GM, Guimarães MDC. Compreensão de informações relativas ao tratamento anti-retroviral entre indivíduos infectados pelo HIV. Cad Saúde Pública 2004; 20:1388-97.

18. Codina-Jané C, Creus MT, Barrueta OI, Sánchez OD, Echevarría OM, Díaz BG. Evaluación de un programa de atención farmacéutica dirigido a mejorar la adherencia al tratamiento antirretroviral. Farm Hosp 2004; 28 Suppl 1:19-26.

19. Hardy H. Adherence to antiretroviral therapy: the emerging role of HIV pharmacotherapy specialists. J Pharm Pract 2005; 18:247-57.

20. Castilho E, Palepu A, Beardsell A, Akagi L, Yip B, Montaner JSG, et al. Outpatient pharmacy care and HIV viral load response among patients on HAART. AIDS Care 2004; 16:446-57.

21. Ministério da Saúde. Assistência farmacêutica. http://www.saude.gov.br (acessado em 09/ Dez/2007).

22. Programa Nacional de DST e AIDS, Secretaria de Vigilância em Saúde, Ministério da Saúde. Plano estratégico do PN-DST/AIDS para 2005. Brasília: Ministério da Saúde; 2005.

23. Grossberg R, Zhang Y, Gross R. A time-to-prescription-refill measure of antiretroviral adherence predicted changes in viral load in HIV. J Clin Epidemiol 2004; 57:1107-10.

24. Kitahata MM, Reed SD, Dillingham PW, Stephen EVR, Young AA, Harrington RD, et al. Pharmacybased assessment of adherence to HAART predicts virologic and immunologic treatment response and clinical progression to AIDS and death. Int J STD AIDS 2004; 15:803-10.

25. Nachega JB, Hislop M, Dowdy DW, Chaisson RE, Regensberg L, Maartens G. Adherence to non-nucleoside reverse transcriptase inhibitor-based HIV therapy and virologic outcomes. Ann Intern Med 2007; 146:564-74.

26. Nachega JB, Hislop M, Dowdy DW, Melane LO, Omer SB, Regensberg L, et al. Adherence to highly active antiretroviral therapy assessed by pharmacy claims predicts survival in HIV-infected South African adults. J Acquir Immune Defic Syndr 2006; 43:78-84.

27. Hogg RS, Heath K, Bangsberg D, Yip B, Press N, O'Shaughnessy MV, et al. Intermittent use of triple-combination therapy is predictive of mortality at baseline and after 1 year of follow-up. AIDS 2002; 16:1051-8

28. Guimarães MDC, Rocha GM, Campos LN, Freitas FMT, Gualberto FAS, Teixeira RAR, et al. Difficulties reported by HIV-infected patients using antiretroviral therapy in Brazil. Clinics 2008; 63:165-72.

29. Rodrigues CS, Guimarães MDC, Acurcio FA, Comini CC. Interrupção do acompanhamento clínico ambulatorial de pacientes infectados pelo HIV. Rev Saúde Pública 2003; 37:183-90.
30. Guimarães MDC, Acurcio FA, Freitas MIF, Bonolo PF, Ceccato MGB, Campos LN, et al. Fatores associados à adesão ao tratamento anti-retroviral (ARV) em indivíduos infectados pelo HIV/AIDS: uma abordagem quantitativa e qualitativa, Belo Horizonte (MG), 2001-2003 (Projeto ATAR). Belo Horizonte: Programa Nacional de DST/AIDS; 2003.

31. Bonolo PF, César CC, Acurcio FA, Ceccato MGB, Menezes-de-Pádua CA, Alvares J, et al. Non-adherence among patients iniciating antiretroviral therapy: a challenge for health professionals in Brazil. AIDS 2005; 19 Suppl 4:5-13.

32. Álvares J. Avaliação do registro diário de medicamentos como instrumento de medida de adesão, Belo Horizonte, 2001-2003 [Dissertação de Mestrado]. Belo Horizonte: Faculdade de Farmácia, Universidade Federal de Minas Gerais; 2004.

33. Hosmer DW, Lemeshow S. Goodness of fit tests for the multiple logistic regression model. Communications in Statistics - Theory and Methods 1980; 9:1043-69.

34. Bomtempo NM. Estudo de fatores de risco para uso irregular do tratamento anti-retroviral, em um serviço público de referência, em Minas Gerais [Dissertação de Mestrado]. Belo Horizonte: Faculdade de Medicina, Universidade Federal de Minas Gerais; 2000

35. Brito AM, Szwarcwald CL, Castilho EA. Fatores associados à interrupção de tratamento anti-retroviral em adultos com AIDS. Rio Grande do Norte, 1999-2002. Rev Assoc Méd Bras 2006; 52:86-92.

36. Martín-Sánchez V, Ortega-Valín L, Pérez-Simón MR, Mostaza-Fernández JL, Urbina-González JJO, Rodríguez-Maria M, et al. Factores predictores de no-adherencia al tratamiento antirretroviral de gran actividad. Enferm Infecc Microbiol Clin 2002; 20:491-7.

37. Ministério da Saúde. Terapia anti-retroviral e saúde pública: um balanço da experiência brasileira. Brasília: Ministério da Saúde; 1999.

38. Lucas GM, Gebo KA, Chaisson RE, Moore RD. Longitudinal assessment of the effects of drug and alcohol abuse on HIV-1 treatment outcomes in an urban clinic. AIDS 2002; 16:767-74.

39. Braithwaite RS, McGinnis KA, Conigliaro J, Maisto SA, Crystal S, Day N, et al. A temporal and doseresponse association between alcohol consumption and medication adherence among veterans in care. Alcohol Clin Exp Res 2005; 29:1190-7.

40. Singh N, Squier C, Sivek C, Wagener M, Hong Nguyen M, Yu VL. Determinants of compliance therapy in patients with human immunodeficiency virus: prospective assessment with implications for enhancing compliance. AIDS Care 1996; 8:261-9.

41. Acurcio FA, Guimarães MD. Utilização de medicamentos por indivíduos HIV positivos: abordagem qualitativa. Rev Saúde Pública 1999; 33:73-84.

42. Yokaichiya CM, Figueiredo WSF, Schraiber LB. Usuários de drogas injetáveis e terapia anti-retroviral: percepções das equipes de farmácia. Rev Saúde Pública 2007; 41 Suppl 2:14-21.

Recebido em 15/Fev/2008

Versão final reapresentada em 29/Jul/2008

Aprovado em 08/Out/2008 